\title{
Scientists in film: an interview with Giuseppe Mussardo
}

\author{
Gian Italo Bischi
}

Published online: 23 July 2014

(C) Centro P.RI.ST.EM, Università Commerciale Luigi Bocconi 2014

\begin{abstract}
Gian Italo Bischi interviews Giuseppe Mussardo, a theoretical physicist and author of several documentary films on important figures in the history of science. The interview sheds light on the importance of the documentary and fiction in popular science, and the kind of work that goes into creating this kind of scientific communication. Also included in a cameo of Prof. Mussardo and a brief sketch of the life of Bruno Pontecorvo, subject of a documentary made by Mussardo in 2013, on the occasion of the centenary of the birth of the Italian physicist, a student of Fermi and member of the 'boys of Via Panisperna', who moved to the Soviet Union in the 1950s.
\end{abstract}

Keywords Popular science · Documentary films · History of science · Giuseppe Mussardo - Bruno Pontecorvo . Subrahmanyan Chandrasekhar · Ludwig Boltzmann . Abdus Salam

Giuseppe Mussardo, a theoretical physicist, author of more than 100 scientific papers in international journals and several books, is also interested in the popularisation of science (see Appendix 1). He has published various articles on the history of science, and is the director of the Interdisciplinary Laboratory of Natural Sciences and Humanities of the Scuola Internazionale Superiore di Studi Avanzati (SISSA) in Trieste, Italy, whose aim is to explore the relationships between scientific culture and that of the humanities, and promote public events and initiatives focussed on science, art, philosophy and

G. I. Bischi $(\bowtie)$

DESP Dipartimento di Economia, Società, Politica, Università di

Urbino, Via Saffi n. 42, 61029 Urbino, Italy

e-mail: gian.bischi@uniurb.it history. He is also the author of four documentary films. The first, 'Boltzmann. Il genio del disordine' (Boltzmann: The genius of disorder), made in 2007, is dedicated to the Viennese physicist Ludwig Boltzmann, known for his fundamental and innovative research on entropy, who committed suicide in Trieste. The next, made in 2009, was 'Chandra. Il viaggio di una stella' (Chandra: The journey of a star), about the Indian physicist Subrahmanyan Chandrasekhar, who was awarded the Nobel Prize for Physics in 1983 for his studies of the structure and evolution of the stars. This was followed, in 2011, by 'Abdus Salam. The Dream of Symmetry', about the Pakistani physicist, winner of the Nobel Prize in 1979 for his contribution to theory of the unified weak and electromagnetic interaction between elementary particles; Salam was the founder of the International Centre for Theoretical Physics in Trieste. Most recently, in 2013, Mussardo made 'Maksimovič. The Story of Bruno Pontecorvo' (Fig. 1), created on the occasion of the centenary of the birth of the Italian physicist, a student of Fermi and member of the famous 'boys of Via Panisperna', who moved to the Soviet Union in the 1950s (see Appendix 2).

In recognition of his work in the popularisation of science, Mussardo received the 'Premio per l'Outreach 2013', awarded by the Italian Physical Society to promote the spread of knowledge of physics and the growth of scientific culture in Italy. The award statement says:

For his noteworthy contributions to the spread of knowledge in physics and the growth of scientific culture among the public at large, in particular with the creation of a series of original film documentaries about great figures in physics.

I interviewed Giuseppe Mussardo on the occasion of the presentation of the documentary about Bruno Pontecorvo. 


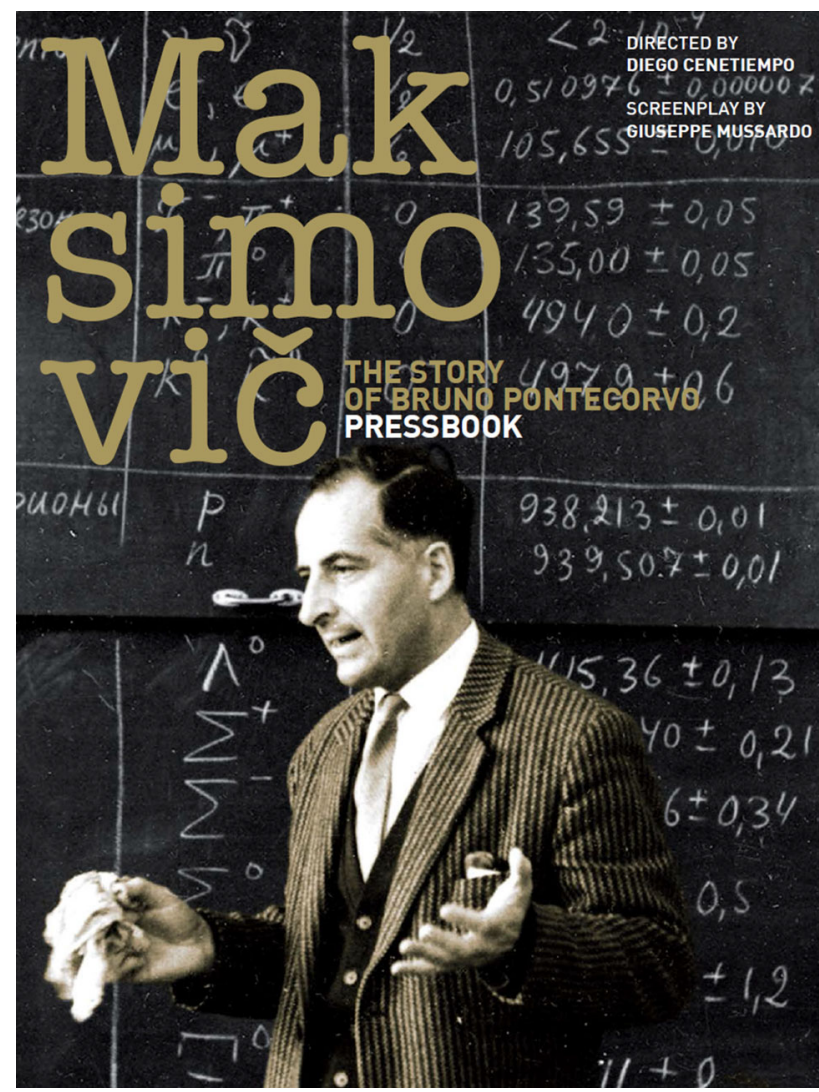

Fig. 1 Poster for the documentary 'Maksimovič. The story of Bruno Pontecorvo'

Gian Italo Bischi (B). A physicist usually selects articles or books as instruments of divulgation. Why did you opt for film documentaries?

Mussardo (M). There are various reasons, not least my passion for photography and cinema. But beyond these interests, it must be said that a documentary film, in contrast to a book or article, forces the author's hand, making him use a language that is necessarily direct, comprised of words, concepts, and ideas that are absolutely concise. This is the great challenge that fascinates me, and lies behind my choice of making documentaries: how is it possible to communicate the concepts that are important (but more often abstruse) in our scientific culture? In doing this, I have learned that it is not necessary to aim at exhaustiveness (the privileged terrain of books, for example). Nevertheless, what I have always tried to do is to be as precise as possible (from a scientific as well as historical point of view) yet without being didactic or boring. It's quite an undertaking!

(B.) What was the first documentary that you made, and where did you get the idea?

(M.) The first documentary was about Ludwig Boltzmann and the arrow of time. To better understand what is behind each of my documentaries, it might be helpful to talk about their basic idea: each documentary deals with a great scientific theme but combines it with a great historic figure. This person plays the same role as that played by Virgil in the Divine Comedy, that is, that of accompanying the spectator along the historic pathways that follow the birth of a great scientific idea; it does this with the help of a fascinating biography. Each documentary can thus be seen in at least three different ways, and there are at least three different types of spectator: (1) those who have scientific knowledge and interests and might be interested in the scientific topic in itself as well as in the various steps that led to its historic maturation; (2) those who are without any science and who must be given the opportunity to enjoy the biographical story, which therefore has to be interesting in itself; and finally (3) the 'ideal spectator', that is, those who are interested in both the history of science (and in the biography of the person) and in the scientific topic itself, and is thus capable of following each scientific phase of the film without problem, and enjoying the narrative plot at the same time.

(B.) Have you gotten the most appreciation from students, colleagues, or non-specialists?

(M.) Based on what I just said, colleagues and students of science are part of the category of 'ideal spectators', and are in fact those who have always shown a great appreciation for the documentaries. But the most satisfying aspect for me is that of having seen the great interest and lively appreciation on the part of those who have never pondered scientific subjects, I mean those who start out by saying, 'In high school I never understood anything about mathematics and physics'.

(B.) Why is it important to tell the stories of scientific personalities outside the circle of scientists?

(M.) It is important because science is an integral part of modern culture. Science, among all else, it bears repeating, is not just pure technology. It is a wrong to confuse science with technology, as often happens. Science has its history, which is as fascinating as that of art, literature and philosophy. The great scientists have opened grand panoramas on the world, equal to the great explorers who extended our geographical confines, and have posed questions of interest to all, such as (to give only a few examples): Why does time always go forward? How did the universe originate? Will it ever end? What is matter made of and what laws is it subject to? These are questions that the great scientists tried to answer thanks to a method, a procedure, in which logic is the master, and principle of authority that holds. The scientific method should be part of everyone's cultural baggage, apart from the knowledge that one may or may not have on various scientific subjects. The scientific method means presenting subjects that are corroborated by logic, supported by experience and not merely by opinions, which may even be contaminated by various ideologies. 
This is what is learned by studying the history of science, a guideline to knowledge of the world and nature.

(B.) Where do you begin making a documentary dedicated to a scientific personality? What kind of preparatory work is there behind it?

(M.) Behind every documentary is about 2 years of intense, detailed work of both writing and research, this last to search the archives for all possible documents or original sources. These might be of different kinds and of different degrees of difficulty to obtain: they go from scientific articles to photographs, to archival materials, to films, personal letters, and so forth. In the last two documentaries about Abdus Salam and Pontecorvo, I was lucky enough to work with Luisa Bonolis, an excellent historian of science, and with Diego Cenetiempo, a brilliant young director. The team worked very well together, and harmonised with the various kinds of activity involved in a documentary: the narrative, the historiographic, and cinematographic. The creation of each documentary is born by following my basic interest in the various characters, an interest that might have been dormant for years but was reawakened through reading, or a chance photo found at a market stall, a newspaper article, or an old library book. Finally at a certain point a more detailed and compelling project takes shape: the research becomes increasingly in-depth, almost obsessive. For the personalities in my documentaries I have an impressive number of final documents.

(B.) In a documentary of yours, how much weight is given to the human side, and how much to the aspects that are strictly scientific, and to which of these two aspects do you want to draw the most attention? How important in the life of a scientist is the historic-social-political context?

(M.) I am of the school of thought that says that, if Planck had not taken the first steps towards quantum mechanics, then some other scientist would surely have introduced the famous 'elementary quantum of action'. This is a distinctive characteristic of science. Its progress is tied more to cognitive thrusts of a vast community than to the peculiarities of an individual. This said however, I find it extremely interesting to understand how the human qualities and historical-biographical events of each individual scientist influenced the development of various branches of science. Sometimes they have drastically influenced the development of history, such as in the case of Fermi, Bethe, Oppenheimer, and others, that is, the nuclear scientists of the Manhattan project. Sometimes instead it is history that overwhelms their lives, as in the case of Bruno Pontecorvo (see Appendix 2). All of these aspects make a narrative interesting and enthralling.

(B.) Two narrative styles are mixed in the documentary about Pontecorvo: the documentary proper, with period films and photographs, and fiction, with actors who play
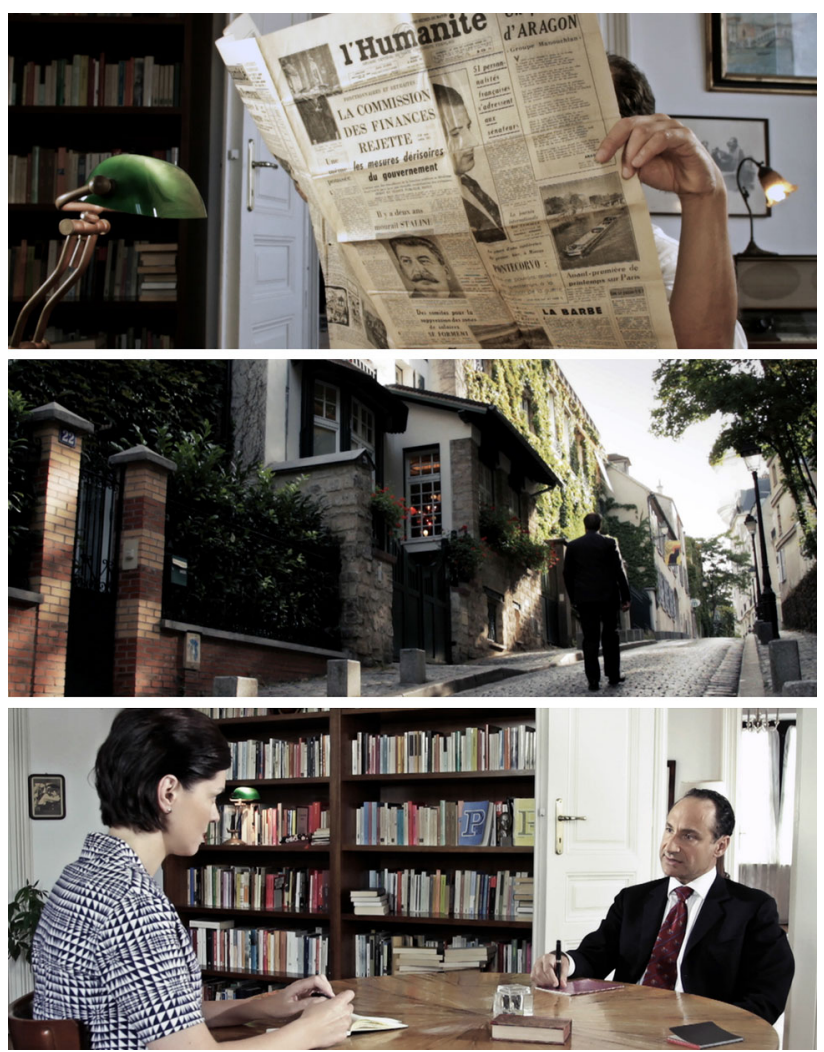

Fig. 2 Scenes from the documentary 'Maksimovič. The story of Bruno Pontecorvo'

the parts of Pontecorvo and the journalist who interviews him. Which of the two styles (fiction and documentary) do you believe to be most effective? Or is a compromise between the two always preferable?

(M.) Without a doubt, for the average spectator (that is, the one without any science) the fiction style is the one that is most effective. But $\mathrm{I}$ am of the opinion that a scientific documentary is something quite different from fiction. In the case of Pontecorvo, for example, even though his story lends itself naturally to film, pure fiction would have the serious defect of putting his greatness as a scientist in the background, of not allowing the fact to emerge that Pontecorvo was the first scientist to understand the secrets of our universe's most elusive particle, the neutrino, a thing of enormous scientific importance [1]. Combining the two narrative languages is difficult, but, to my mind, when such a synthesis succeeds, it is what renders the final work most interesting (Fig. 2).

(B.) Which of your documentaries has given you the most satisfaction, and which of the characters are you most fond of?

(M.) That's difficult for me to answer. All four of my documentaries have given me great satisfaction and I am tied to all four characters, although for different reasons: Boltzmann because my area of research is in statistical 


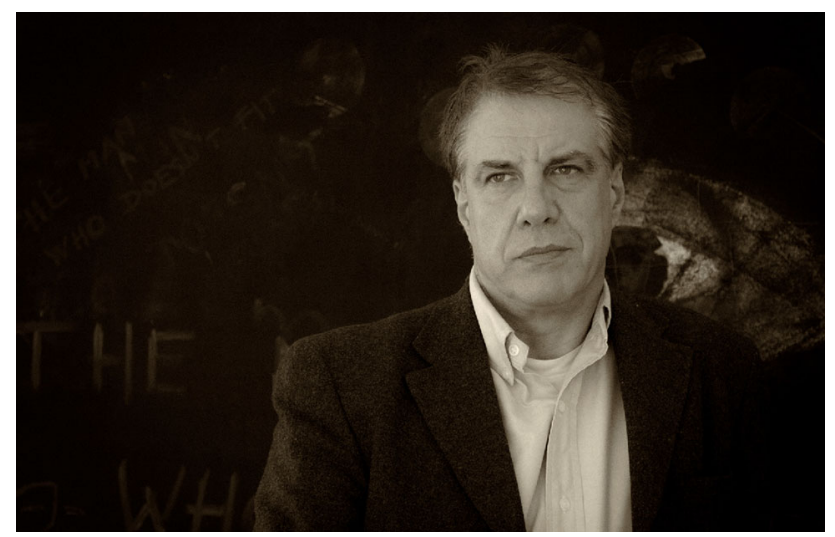

Fig. 3 Giuseppe Musssardo

physics and because his is a very Mitteleuropean story; Chandrasekhar because his is a story of a great discovery made at an extremely young age during a long sea journey, but long scoffed at, out of racism or academic bullying, by the British 'university barons' of the day; Salam because his name is associated with the most advanced theory we know about the ultimate constituents of matter, but above all with his thirst for knowledge and his idea of creating a common heritage for all humanity, even for the poorest people on the planet. And then there is Pontecorvo, because his is one of the most fascinating stories ever: a person who disappears for 5 years, to reappear on the other side of the Iron Curtain during the dark years of the 1950s, an incredible, really beautiful story.

(B.) Do you already have an idea in mind for the next documentary/fiction?

(M.) We'll talk about that again in a few years.

\section{Appendix 1: Giuseppe Mussardo}

Giuseppe Mussardo is Professor of theoretical physics at the Scuola Internazionale Superiore di Studi Avanzati (SISSA) in Trieste, where he coordinates the statistical physics group, which he founded in 2000, and he is also the director of the Ph.D. program associated with it (Fig. 3). He is editor-in-chief of the scientific journal Journal of Statistical Physics and Applications (JSTAT) and a member of the editorial board of Nuclear Physics $B$. He is the author of the monographs Statistical Field Theory. An Introduction to Exactly Solved Models in Statistical Physics (Oxford University Press, 2009) and Il Modello di Ising. Introduzione alla Teoria dei Campi e delle Transizioni di Fase (Bollati-Boringhieri, 2006). Due out soon is his book of popular science, L'alfabeto della scienza.
Appendix 2: Brief biography of Bruno Pontecorvo

I, like a river,

Was diverted by this stern epoch. They gave me a substitute life. It flowed Into a different channel, past the other one

And I do not know my own banks.

From "I, like a river" by Anna Achmatova [2]

Bruno Pontecorvo was born in Marina di Pisa in 1913, to a wealthy family of Jewish origin. He was the older brother of Gillo (a famous director) and younger brother of Guido (a well-known biologist-geneticist) [3]. After 2 years of engineering in Pisa, at the age of only 18 he enrolled in the third year of physics at the University of Rome, where he became a student of Enrico Fermi's. From 1934 to 1936 he was part of the 'boys of Via Panisperna', collaborating with them on the famous 1934 experiment on slow neutrons, which marked the beginning of research on nuclear fission and its applications. He was the youngest and newest member of the group, and for this reason was nicknamed il cucciolo, 'the pup' (the others had nicknames too; Fermi was 'the Pope', Ettore Majorana was 'the Great Inquisitor', and so on).

Thanks to a scholarship from the Ministry for Public Instruction, in 1936 he moved to Paris, where he worked at the Institut du Radium with Irène Curie and Frédéric Joliot, and formed friendships with many other scientists. During his stay in Paris he came to embrace Marxist ideology. In August 1940, after the Nazi invasion of Paris, he fled to the United States, where he worked for Wells Survey in Tulsa, Oklahoma, a petroleum company. There he perfected a technique of well-logging, ${ }^{1}$ based on the properties of neutrons. This was the first practical application of the discovery made in Rome with Fermi of the properties of slow neutrons. In 1943 he was called to take part in the theoretical research near Montreal, in Canada, where he was involved in the study of cosmic rays, and in particular, of neutrinos and muon decay. In 1948, after being granted British citizenship, he moved to the Atomic Energy Research Establishment (AERE) near Harwell, Oxfordshire.

On 31 August 1950, during a holiday in Italy, without saying anything to friends or relatives, he and his whole family (his wife, and three children) left Rome for Stockholm, went from there to Helsinki, and then to Russia, where he became a Russian citizen with the name Bruno Maksimovič Pontekorvo (Бруно Максимович Понтекорво).

At first his sudden disappearance raised fears of a new case like that of Majorana, ${ }^{2}$ then wreaked havoc within

\footnotetext{
${ }^{1}$ Well-logging is the practice of making a detailed record (a 'well $\log$ ') of the geologic formations penetrated by a borehole.

2 Ettore Majorana's mysterious and sudden disappearance in 1938 has never been explained.
} 
Western national security agencies, who were worried about the possible transmission of atomic secrets during the height of the Cold War [4].

There followed a period of intense work in the laboratories of Dubna, where the Soviets had installed an important laboratory for atomic research on high-energy particles and in particular on muon decay and neutrinos. Pontecorvo was awarded the Stalin Prize in 1954 for his research, and became a member of the prestigious Russian Academy of Sciences in 1958. This period saw the maturation of his fundamental studies on elementary particle physics and later, in astrophysics, with important contributions to neutrino physics and the investigation of solar neutrinos, studies that led to his being awarded the Lenin Prize in 1963 for his research on neutrinos and weak interactions.

Although Pontecorvo had been welcomed to the Soviet Union with acclaim and honour, and provided with all that was necessary for his work and a comfortable life for his family, he was forced into isolation, deprived on contact with the outside work, with no possibility of either leaving the USSR or publishing his work in Western journals. Only in 1955 was he permitted to appear in public, on the occasion of a press conference where he explained to the world his reasons for abandoning Western society and his belief in communism. It was not until many years later that he was able to travel abroad and visit Italy (the first time in 1978 on the occasion of the physicist Edoardo Amaldi's seventieth birthday). He returned to Italy on other occasions as well, but he continued to live in Russia even after the fall of communism.

Afflicted with Parkinson's, he died in Dubna in 1993. As he expressly desired, half of his ashes are buried in the Dubna cemetery, and half in the non-Catholic cemetery in Rome.

Translated from the Italian by Kim Williams.

\section{References}

1. Bonolis, L.: Bruno Pontecorvo, from slow neutrons to oscillating neutrinos. Am. J. Phys. 73, 487-499 (2005)

2. Harrington, A.: The Poetry of Anna Achmatova: Living in a Different Mirror. Anthem Press, London (2006)

3. Mafai, M.: Il lungo freddo. Storia di Bruno Pontecorvo, lo scienziato che scelse l'URSS, Milano, Mondadori (1992)

4. Turchetti, S.: Il caso Pontecorvo. Fisica nucleare, politica e servizi di sicurezza nella guerra fredda, Milano, Sironi (2007)

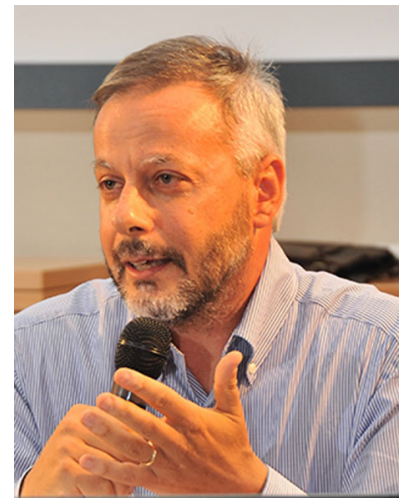

the Lettera Matematica.
Gian Italo Bischi is a professor of mathematical methods for economics and finance at the University of Urbino. He has published articles and books on dynamic models and their applications to the description of complex systems. He also works in popular science, in particular on the connection between mathematics and the other fields of knowledge, in the context of the activities of the Centro PRISTEM. $\mathrm{He}$ is a member of the editorial board of 